\title{
氮添加对亚热带次生常绿阔叶林扁刺栲细根生物 量、寿命和形态的短期影响
}

陈冠陶 ${ }^{1}$ 彭 勇 ${ }^{1}$ 郑 军 ${ }^{2}$ 李 顺 $^{1}$ 彭天驰 ${ }^{1}$ 邱细容 ${ }^{1}$ 涂利华 $1^{*}$

${ }^{1}$ 四川农业大学林学院, 成都 $611130{ }^{2}$ 四川省遂宁市林业局, 四川遂宁 629000

摘 要 细根在植物的养分获取和生态系统物质循环中有重要的作用, 日益增加的氮沉降改变了土壤 $\mathrm{pH}$ 值和养分可利用性, 潜在地影响着细根特性。为揭示氮沉降增加对木本植物细根寿命和形态的影响, 于2013年5月至2015年9月在亚热带次生常绿 阔叶林中开展每月一次的氮添加试验, 共设对照 $\left(\mathrm{CK}, 0 \mathrm{~g} \cdot \mathrm{m}^{-2} \cdot \mathrm{a}^{-1}\right)$ 、低氮 $\left(\mathrm{LN}, 5 \mathrm{~g} \cdot \mathrm{m}^{-2} \cdot \mathrm{a}^{-1}\right)$ 、高氮 $\left(\mathrm{HN}, 15 \mathrm{~g} \cdot \mathrm{m}^{-2} \cdot \mathrm{a}^{-1}\right) 3$ 个水平。 以该林分建群种扁刺栲(Castanopsis platyacantha)为研究对象，于2014年4月至2015年9月，每月采用微根管技术获取根系影 像, 室内分析后得到各样地的扁刺栲细根生物量、寿命和形态指标。2014年11月取 0-10 cm土壤分析土壤 $\mathrm{pH}$ 值、铵态氮和硝 态氮含量。结果表明: 扁刺栲 $0-45 \mathrm{~cm}$ 土层细根生物量为 $128.30 \mathrm{~g} \cdot \mathrm{m}^{-3}$, 细根平均寿命范围为113-186天; 氮添加处理未显著影 响扁刺栲0-45 cm土层细根寿命和生物量; 低氮处理显著降低了 0-15 cm土层细根根段表面积; 高氮处理显著降低了表层土 壤 $\mathrm{pH}$ 值。研究表明短期氮添加通过影响该次生林土壤无机氮含量, 导致表层土壤酸化, 对表层土壤扁刺栲细根的形态特征造 成一定的影响; 短期氮添加对扁刺栲细根生物量、寿命和深层细根的形态未产生显著影响。

关键词 氮沉降; 细根生物量; 细根寿命; 细根形态; 微根管

引用格式: 陈冠陶, 彭勇, 郑军, 李顺, 彭天驰, 邱细容, 涂利华 (2017). 氮添加对亚热带次生常绿阔叶林扁刺栲细根生物量、寿命和形态的短期影响. 植物生态学报, 41, 1041-1050. doi: 10.17521/cjpe.2016.0317

\section{Effects of short-term nitrogen addition on fine root biomass, lifespan and morphology of Cas- tanopsis platyacantha in a subtropical secondary evergreen broad-leaved forest}

CHEN Guan-Tao ${ }^{1}$, PENG Yong ${ }^{1}$, ZHENG Jun ${ }^{2}$, LI Shun ${ }^{1}$, PENG Tian-Chi ${ }^{1}$, QIU Xi-Rong ${ }^{1}$, and TU Li-Hua ${ }^{1 *}$

${ }^{1}$ College of Forest, Sichuan Agricultural University, Chengdu 611130, China; and ${ }^{2}$ Suining Forestry Bureau, Suining, Sichuan 629000, China

\section{Abstract}

Aims Fine roots are the principal parts for plant nutrients acquisition and play an important role in the underground ecosystem. Increased nitrogen $(\mathrm{N})$ deposition has changed the soil environment and thus has a potential influence on fine roots. The purpose of this study is to reveal the effect of $\mathrm{N}$ deposition on biomass, lifespan and morphology of fine root.

Methods A field N addition experiment was conducted in a secondary broad-leaved forest in subtropical China from May 2013 to September 2015. Three levels of N treatments: CK (no N added), LN (5 $\mathrm{g} \cdot \mathrm{m}^{-2} \cdot \mathrm{a}^{-1}$ ), and HN $\left(15 \mathrm{~g} \cdot \mathrm{m}^{-2} \cdot \mathrm{a}^{-1}\right)$ were applied monthly. Responses of fine root biomass, lifespan, and morphology of Castanopsis platyacantha to $\mathrm{N}$ addition were analyzed by using a minirhizotron image system from April 2014 to September 2015. Surface soil sample $(0-10 \mathrm{~cm})$ was collected in November 2014 and soil pH value, and concentrations of $\mathrm{NH}_{4}^{+}-\mathrm{N}$ and $\mathrm{NO}_{3}^{-}-\mathrm{N}$ were measured.

Important findings The biomass and average lifespan of the fine roots of C. platyacantha were $128.30 \mathrm{~g} \cdot \mathrm{m}^{-3}$ and 113-186 days, respectively, in 0-45 cm soil layer. Nitrogen addition had no significant effect on either fine root biomass or lifespan in 0-45 cm soil layer. However, LN treatment significantly decreased C. platyacantha root superficial area in $0-15 \mathrm{~cm}$ soil layer. $\mathrm{HN}$ treatment significantly decreased soil $\mathrm{pH}$ value. Our study indicated that short-term $\mathrm{N}$ addition influences soil inorganic $\mathrm{N}$ concentration and thus decreased $\mathrm{pH}$ value in surface soil, and thereafter affect fine root morphology. Short-term N addition, however, did not affect the fine root biomass, lifespan and morphology in subsoil.

Key words nitrogen deposition; fine root biomass; fine root lifespan; fine root morphology; minirhizotron

收稿日期Received: 2016-10-11 接受日期Accepted: 2017-08-31

* 通信作者Author for correspondence (E-mail: tulhsicau@163.com) 
Citation: Chen GT, Peng Y, Zheng J, Li S, Peng TC, Qiu XR, Tu LH (2017). Effects of short-term nitrogen addition on fine root biomass, lifespan and morphology of Castanopsis platyacantha in a subtropical secondary evergreen broad-leaved forest. Chinese Journal of Plant Ecology, 41, 1041-1050. doi: 10.17521/cjpe.2016.0317

细根占陆地生态系统植物总生物量的比例小于 $2 \%$, 但由于细根周转快速, 消耗了 $20 \%-30 \%$ 的年净 初级生产力(Jackson et al., 1997; Poorter et al., 2011), 因此, 细根周转推动着陆地生态系统碳和养分循 环。影响细根周转速率的主导因子是细根寿命 (Majdi et al., 2005)。根系是植物和土壤交互的界面, 对土壤环境变化高度敏感，根系不断调节自身特性 以适应土壤环境变化, 进而支持植物正常生长

(Eissenstat \&Yanai, 1997; Eissenstat et al., 2000; Wurzburger \& Wright, 2015)。这类调节涉及生物量、 形态、生理过程等多方面, 且相互紧密联系, 进而影 响包括根系周转在内的诸多生态系统过程(Bardgett et al., 2014)。

植物主要通过根系获取维持自身生长发育的多 种元素, 其中, 氮元素对植物的意义最为重大 (Nacry et al., 2013)。大多数陆地生态系统的净初级 生产力受到氮元素的限制 (LeBauer \& Treseder, 2008)。根据“成本-效益”理论, 植物根系总是以尽可 能少的碳消耗来满足植物对氮素的需求(Eissenstat \& Yanai, 1997)。因此, 土壤氮素可利用性对根系寿 命和形态具有重要影响(Eissenstat et al., 2000), 这 在氮沉降梯度研究和元素添加试验中得到了广泛证 明(Ostonen et al., 2007; Li et al., 2015)。

近几十年来，由于工农业发展和化石燃料的大 量燃烧导致氮沉降在全球范围增加, 人类活动极大 地干扰了全球氮循环(Galloway \& Cowling, 2002; Galloway et al., 2004), 对森林生态系统物质循环造 成了潜在的巨大影响(吕超群等, 2007)。大量研究表 明, 日益增加的氮沉降会增加土壤氮素可利用性, 多数情况会下提高植被地上部分生产力(Bedison \& Mcneil, 2009)。然而森林植物根系对土壤氮素增加 的响应方向和程度却存在很大的不确定性。有研究 表明氮添加增加了根系寿命, 减缓了细根周转速率 (Jourdan et al., 2008; 于水强等, 2009; Mei et al., 2010), 但也有研究观察到与之相反的结果(Johnson et al., 2000; Wang et al. 2012)。现有的少量氮添加对 森林植物根系影响的相关研究往往在人工林中开展 (于水强等, 2009)。人工林林分简单, 为根系研究提 供了很大便利(史建伟等, 2006)。然而作为全球森林
主体的天然林(Chirici et al., 2013), 往往与人工林在 土壤特性、林分结构、稳定性、物种多样性等方面 存在极大的差异(Hou et al., 2010; 李丹等, 2011)。因 此, 在天然林中开展相关研究对于更全面地了解氮 沉降对森林生态系统的影响具有重要意义。

根系生长于地下, 不易接近和直接观察, 对于 根系的认识很大程度上受限于研究手段。传统挖掘 法、土钻法、内生长法研究根系, 往往费时费力, 对 根系破坏严重, 且不能连续观察, 很难实现对根寿 命的准确测定(李俊英等, 2007)。近年来, 微根管技 术以其特有的原位性、非破坏性等特点在根系研究 中得到快速发展。通过微根管技术能直接观察单个 根段从出生到死亡的全过程(Chen \& Brassard, 2013; Balogianni et al., 2016), 在细根生产、寿命、周转、 细根生物量的估算等方面应用越来越广泛, 并得到 普遍认可(Addo-Danso et al., 2016)。

为此, 本研究于 2013年5月到2015年9月在四川 瓦屋山次生常绿阔叶林中开展每月一次的氮添加试 验，并在氮添加处理1年后(2014年4月)，每月使用 微根管技术对该林分建群种扁刺栲 (Castanopsis platyacantha)根系影像进行收集，连续收集18个月， 研究扁刺栲细根生物量、寿命和形态对短期氮添加 的响应，以期为深入理解氮沉降对森林地下生态系 统过程的影响提供基础数据和参考。

\section{1 材料和方法}

\section{1 研究区概况}

试验地位于四川洪雅县南部的瓦屋山国家森林 公园中山部的木姜杜林班 $\left(29.54^{\circ} \mathrm{N}, 103.26^{\circ} \mathrm{E}\right)$, 海 拔 $1600 \mathrm{~m}$, 属于中亚热带季风性湿润气候。年平均 气温 10-14 ${ }^{\circ} \mathrm{C}$, 年日照时间不足 $800 \mathrm{~h}$, 年降水量 $2398 \mathrm{~mm}$ ，降水日199-226天，年平均相对空气湿 度85\%-90\%。该区域植被在1956年遭到砍伐破坏， 遗留下一些幼树幼苗, 后基本无进一步的干扰破坏 而得以自然恢复。目前已形成次生常绿阔叶成林, 优势种为扁刺栲和中华木荷(Schima sinensis), 主要 伴生树种为硬壳柯 (Lithocarpus hancei)、野梧桐 (Mallotus japonicus)、小叶青冈 (Cyclobalanopsis myrsinifolia) 等阔叶树种。该区域成土母质是晚古生 
界二叠纪至元古界的砂岩、玄武岩、花岗岩等古老 岩层风化的残积物, 土壤类型为山地黄壤, 土壤厚 约 $1 \mathrm{~m}$, 其中腐殖质层厚约 $10 \mathrm{~cm}$ 。土壤基本理化性 质见表1。

\section{2 试验设计}

2012年10月, 根据典型性和代表性原则在木姜 杜林班设置9块 $20 \mathrm{~m} \times 20 \mathrm{~m}$ 的样地。各样地坡向均 为北向, 坡度 $0-5^{\circ}$, 样地间缓冲距离大于 $20 \mathrm{~m}$ 。使用 $\mathrm{NH}_{4} \mathrm{NO}_{3}$ 进行氮添加处理, 共设3个水平: 对照(CK, $\left.0 \mathrm{~g} \cdot \mathrm{m}^{-2} \cdot \mathrm{a}^{-1}\right)$, 低氮 $\left(\mathrm{LN}, 5 \mathrm{~g} \cdot \mathrm{m}^{-2} \cdot \mathrm{a}^{-1}\right)$, 高氮 $(\mathrm{HN}$, $\left.15 \mathrm{~g} \cdot \mathrm{m}^{-2} \cdot \mathrm{a}^{-1}\right)$, 每个处理设置3个重复。将各处理的 $\mathrm{NH}_{4} \mathrm{NO}_{3}$ 年添加量均分为 12 份, 即得到各处理每月 添加量, 从2013年5月到2015年9月, 每月下旬对各 样地进行定量氮添加处理。具体方法是将各处理所 需的 $\mathrm{NH}_{4} \mathrm{NO}_{3}$ 溶解于 $10 \mathrm{~L}$ 自来水中, 用喷雾器在该 样方中来回均匀喷酒, CK只喷酒等量的自来水。

\section{3 微根管安装}

2013年12月初, 于各样地内选择3株胸径大致 相同(25 cm左右)、生长较好、相对独立的扁刺栲作 为细根动态观测的目标树。在树干周围 $1 / 2$ 冠幅的位 置(到树干基部的距离约 $1 \mathrm{~m}$ )对称地安装两根由有 机玻璃制成的微根管(Tingey et al., 2003)。微根管内 径为 $6.4 \mathrm{~cm}$, 长度为 $1 \mathrm{~m}$ 。具体安装过程为: 先用外 径略大于微根管外径的特制土钻朝向树干基部与垂 直方向成 $45^{\circ}$ 角钻一个通直的洞, 深度约为 $80 \mathrm{~cm}$ (换算成垂直深度约为 $56 \mathrm{~cm}$ ), 然后沿洞将微根管插 入土壤中, 插入深度 $80 \mathrm{~cm}$ 。露出地表的微根管部分 先用黑色胶布缠绕, 以防光线透入, 然后再用白色 胶布覆盖, 以防吸热, 最后在顶端加盖密封, 以防 灰尘、水或降雨和其他物体落入管中。在每根管的 外壁刻划一条细线, 以保证每次取样时都以该位置 为起点进行影像数据收集。

\section{4 图像采集和土样根样收集}

从 2014 年 4 月到 2015 年 9 月, 每月下旬采用
CI-600 扫描仪(CID Inc., Vancouver, Washington, USA)进行根系影像收集。每次扫描时, 从地表开始 按 $20 \mathrm{~cm}$ (换算成垂直深度约为 $15 \mathrm{~cm}$ )的间距每管收 集3张图片。即分别获取0-15 cm土层(I层)、15-30 cm 土层(II层)、30-45 cm 土层(III层)的根系图片。每次 的影像收集工作在 1 天内完成。在观察期间共收集到 了2 916张图片(3层× 6管× 9样地× 18次)。2014年11 月(氮处理 1.5 年后), 在每个样地内随机选取 5 个采 样点, 使用土钻钻取 0-10 cm层(腐殖质层)土壤样品 充分混合后带回实验室进行养分测定。为了计算扁 刺栲细根比根长 $(S R L)$ 以计算细根生物量, 于 2016 年4月用土块法获取每个样地的扁刺栲根系, 具体操 作为: 在每个样地的每株目标树基部50-150 $\mathrm{cm}$ 的 圆环区域内用自制取样器挖取一个 $30 \mathrm{~cm} \times 30 \mathrm{~cm} \times$ $20 \mathrm{~cm}$ (长 $\times$ 宽 $\times$ 深)大小的土块; 由于试验区域土壤 疏松, 在样地内将土块中的根系小心挑出, 将土壤 分层回填到挖取的区域; 取出的根系存放于低温保 温箱内, 迅速带回实验室; 用自来水冲洗掉附着在根 系表面较多的土壤, 再用低温去离子水将根系完全 清洗干净, 装袋编号后冷藏储存以保持其活性。

\section{5 图像分析和土样及比根长测定}

微根管影像带回实验室后, 使用Adobe Photoshop CS6软件调节图片亮度和对比度, 并且对每段 根系进行编号并记录每张图片的根段数量。用根系 分析系统WinRHIZOTron MF 2012b 软件对每个影 像中的根系长度、表面积、直径进行量化, 以及根 系类型的区分，由于扁刺栲新生根表皮呈白色，之 后很快变为红色, 由此可以将其与其他树种根系进 行区分, 并且因此将白色和红色根定义为活根, 黑 色的根、皮层脱落或表皮褶皱的根定义为死根; 另 外, 根系在没表现出来死亡形态时的突然消失, 也 定义为死根。根系寿命定义为第一次在影像中出现 时刻到第一次被定义为死亡或消失的时间。

土壤样品带回实验室后挑出土壤中的杂质, 过

表1 瓦屋山次生常绿阔叶林土壤理化性质(平均值土标准误差)

Table 1 Soil physicochemical properties in a secondary evergreen broad-leaved forest, Wawu Mountain (mean $\pm S E$ )

\begin{tabular}{ccccccc}
\hline $\begin{array}{c}\text { 土层深度 } \\
\text { Soil depth }(\mathrm{cm})\end{array}$ & $\mathrm{pH}$ & $\begin{array}{c}\text { 土壤容重 } \\
\text { Soil bulk density }\left(\mathrm{g} \cdot \mathrm{cm}^{-3}\right)\end{array}$ & $\begin{array}{c}\text { 有机碳含量 } \\
\text { Organic carbon content } \\
\left(\mathrm{g} \cdot \mathrm{kg}^{-1}\right)\end{array}$ & $\begin{array}{c}\text { 全氮含量 } \\
\text { Total nitrogen content } \\
\left(\mathrm{g} \cdot \mathrm{kg}^{-1}\right)\end{array}$ & $\begin{array}{c}\text { 全磷含量 } \\
\text { Total phosphorus } \\
\text { content }\left(\mathrm{g} \cdot \mathrm{kg}^{-1}\right)\end{array}$ & $\begin{array}{c}\text { 全钾含量 } \\
\text { Total potassium content } \\
\left(\mathrm{g} \cdot \mathrm{kg}^{-1}\right)\end{array}$ \\
\hline $0-10$ & $3.19 \pm 0.03$ & $0.41 \pm 0.03$ & $121.9 \pm 11.7$ & $5.80 \pm 0.30$ & $0.50 \pm 0.01$ & $13.89 \pm 0.92$ \\
$10-40$ & $3.76 \pm 0.03$ & $0.66 \pm 0.02$ & $26.6 \pm 2.5$ & $1.51 \pm 0.08$ & $0.26 \pm 0.03$ & $0.18 \pm 0.01$ \\
$40-70$ & $3.97 \pm 0.02$ & $0.89 \pm 0.03$ & $12.6 \pm 1.2$ & $0.82 \pm 0.05$ & $17.07 \pm 0.82$ \\
$70-100$ & $4.03 \pm 0.02$ & $0.99 \pm 0.03$ & $7.8 \pm 0.8$ & $0.57 \pm 0.04$ & $0.21 \pm 0.01$ \\
\hline
\end{tabular}

取土样时间为2012年11月28日。

Soil sampled on November 28th, 2012. 
$2 \mathrm{~mm}$ 笁后冷藏保存, 测定土壤 $\mathrm{pH}$ 值、铵态氮 $\left(\mathrm{NH}_{4}^{+}-\mathrm{N}\right)$ 含量、硝态氮 $\left(\mathrm{NO}_{3}^{-}-\mathrm{N}\right)$ 含量, 取部分鲜土风 干后过 $0.25 \mathrm{~mm}$ 篎用于测定土壤全氮(TN)含量。使 用电位法(LY/T 1239-1999)测定土壤pH值, TN用半 微量凯氏法(LY/T 1228-1999)测定, $\mathrm{NO}_{3}^{-}-\mathrm{N}$ 用紫外分 光光度法(宋歌等, 2007)测定, $\mathrm{NH}_{4}^{+}-\mathrm{N}$ 用 $2 \mathrm{~mol} \cdot \mathrm{L}^{-1}$ 的 KCl浸提-靛酚蓝比色法(鲍士旦, 2000)测定。土块法 获取的扁刺栲根系清洗干净, 挑除死根后取直径 $<1$ $\mathrm{mm}$ 细根, 使用 Epson数字化扫描仪 (Expression 10000XL, Epson Electronics, San Jose, USA)扫描。用 WinRHIZO (pro 2007b)根系图像分析软件对根扫描 图像进行根长的定量分析。扫描分析完成后将细根 在65 ${ }^{\circ} \mathrm{C}$ 烘箱中烘干至恒质量, 记录干质量。计算不 同氮处理下的比根长 $(S R L), S R L=$ 根长/干质量。

\section{6 数据处理和分析}

\subsection{1 根系寿命}

建立根系寿命数据库, 将影像中相连但不存在 分叉的一段根系(即根段)作为统计的基本单位。每 一段根系都赋予指定ID号, 按照土壤层次、氮添加 水平分别归类。在Excel中统计各根段的存活天数, 使用Kaplan-Meier方法进行生存分析, 估算细根平 均寿命及中值寿命(即存活率达到 $50 \%$ 时所用的时 间), 并做存活曲线; 使用对数秩检验(log-rank test) 比较不同氮处理对存活曲线的影响。

\subsection{2 细根生物量}

参考吴伊波等(2014)的计算方法, 估算单位土 壤体积扁刺栲细根生物量。

首先, 利用每张影像中的根系长度, 计算单位 土壤体积的根长密度 $\left(R L D, \mathrm{~mm} \cdot \mathrm{cm}^{-3}\right)$ 。计算公式 如下:

$$
R L D=R L /(A \times D O F)
$$

式中, $R L(\mathrm{~mm})$ 为每张影像中的根系长度; $A\left(\mathrm{~cm}^{2}\right)$ 为 影像的面积; $D O F(\mathrm{~cm})$ 为微根管观察可代表的土壤
距离, 本研究中取 $0.25 \mathrm{~cm}$ 。

然后, 通过 $S R L\left(\mathrm{~m}^{-} \mathrm{g}^{-1}\right)$ 将 $R L D$ 转化为单位土壤 体积的细根生物量 $\left(R B D, \mathrm{~g} \cdot \mathrm{m}^{-3}\right)$ 。

$$
R B D=R L D / S R L \times 1000
$$

式中, $S R L$ 为利用土块法取样测定的直径小于 $1 \mathrm{~mm}$ 的扁刺栲细根比根长。对照处理 $S R L$ 为 $10.0 \mathrm{~g} \cdot \mathrm{m}^{-3}$, 低 氮处理 $S R L$ 为 $10.6 \mathrm{~g} \cdot \mathrm{m}^{-3}$, 高氮处理 $S R L$ 为 $9.4 \mathrm{~g} \cdot \mathrm{m}^{-3}$ 。

\subsection{3 根段形态}

影像分析过程中发现, 影像中的根系绝大多数 属于扁刺栲的 1 级或 2 级细根, 因此我们使用根段为 单位来量化根系的形态特征。利用每张影像中的根 系长度 $(R L, \mathrm{~cm})$ 、根系表面积 $\left(R A, \mathrm{~cm}^{2}\right)$ 和根段数量 $(N)$ 计算根段长 $(R S L, \mathrm{~cm})$ 和根段表面积 $\left(R S A, \mathrm{~cm}^{2}\right)$ 。计算 公式如下

$$
\begin{aligned}
& R S L=R L / N \\
& R S A=R A / N
\end{aligned}
$$

\subsection{4 统计分析}

计算观察期内各个指标的样地平均值, 并使用 平均值进行统计分析。使用单因素方差分析和 $L S D$ 多重比较 $(\alpha=0.05)$ 检验氮添加处理对土壤 $\mathrm{pH}$ 值、 $\mathrm{TN} 、 \mathrm{NO}_{3}^{-}-\mathrm{N} 、 \mathrm{NH}_{4}^{+}-\mathrm{N}$ 、根段数量、根段长、根段表 面积、根直径和生物量影响的显著性。对 $0-10 \mathrm{~cm}$ 土壤养分指标和 $\mathrm{I}$ 土层根形态指标及中值寿命数据 进行Pearson相关分析(两尾, $\alpha=0.05, n=9$ )。所有统 计分析均在SPSS 20.0 (IBM SPSS, Chicago, USA)软 件中执行。

\section{2 结果和分析}

\section{1 氮添加对土壤化学指标的影响}

与CK相比, HN处理显著降低了土壤 $\mathrm{pH}$ 值 $(p<$ 0.05)。土壤 $\mathrm{TN} 、 \mathrm{NO}_{3}^{-}-\mathrm{N}$ 含量均表现出随氮添加梯度 增加而增加的趋势, 但未达到统计学显著水平(表 2)。相关分析结果表明土壤 $\mathrm{pH}$ 值与 $\mathrm{NH}_{4}^{+}-\mathrm{N} 、 \mathrm{NO}_{3}^{-}-\mathrm{N}$

表2 氮添加对表层土壤 (0-10 cm)化学养分和 $\mathrm{pH}$ 值的影响(平均值土标准误差)

Table 2 The effect of $\mathrm{N}$ addition on surface soil $(0-10 \mathrm{~cm})$ chemical element and $\mathrm{pH}$ value (mean $\pm S E$ )

\begin{tabular}{lcccc}
\hline & $\mathrm{pH}$ & $\mathrm{TN}\left(\mathrm{g} \cdot \mathrm{kg}^{-1}\right)$ & $\mathrm{NH}_{4}^{+}-\mathrm{N}\left(\mathrm{mg} \cdot \mathrm{kg}^{-1}\right)$ & $\mathrm{NO}_{3}^{-}-\mathrm{N}^{\left(\mathrm{mg} \cdot \mathrm{kg}^{-1}\right)}$ \\
\hline $\mathrm{CK}$ & $3.99 \pm 0.05^{\mathrm{a}}$ & $6.62 \pm 0.50$ & $25.62 \pm 5.79$ & $25.47 \pm 3.90$ \\
$\mathrm{LN}$ & $3.82 \pm 0.02^{\mathrm{ab}}$ & $7.19 \pm 0.57$ & $21.63 \pm 6.02$ & $26.27 \pm 4.65$ \\
$\mathrm{HN}$ & $3.78 \pm 0.08^{\mathrm{b}}$ & $7.23 \pm 0.35$ & $40.15 \pm 12.39$ & $36.69 \pm 4.61$ \\
单因素方差分析 & $p=0.04$ & $p=0.62$ & $p=0.33$ & $p=0.21$ \\
One-way ANOVA analysis & & & & \\
\hline
\end{tabular}

同列的不同字母表示氮添加处理在 0.05 水平下差异显著。CK, 对照 $\left(0 \mathrm{~g} \cdot \mathrm{m}^{-2} \cdot \mathrm{a}^{-1}\right) ; \mathrm{LN}$, 低氮 $\left(5 \mathrm{~g} \cdot \mathrm{m}^{-2} \cdot \mathrm{a}^{-1}\right) ; \mathrm{HN}$, 高氮 $\left(15 \mathrm{~g} \cdot \mathrm{m}^{-2} \cdot \mathrm{a}^{-1}\right)$ 。

Different letters with the same column indicate significant differences among $\mathrm{N}$ addition treatments at 0.05 levels. CK, control $\left(0 \mathrm{~g} \cdot \mathrm{m}^{-2} \cdot \mathrm{a}^{-1}\right)$; $\mathrm{LN}$, low nitrogen $(5$ $\left.\mathrm{g} \cdot \mathrm{m}^{-2} \cdot \mathrm{a}^{-1}\right)$; HN, high nitrogen $\left(15 \mathrm{~g} \cdot \mathrm{m}^{-2} \cdot \mathrm{a}^{-1}\right)$.

www.plant-ecology.com 
含量呈显著负相关关系(表3)。

\section{2 氮添加对扁刺栲细根生物量的影响}

扁刺栲细根 $(<1 \mathrm{~mm})$ 在I、II和III土层生物量分 别是 $65.01 \mathrm{~g} \cdot \mathrm{m}^{-3} 、 40.79 \mathrm{~g} \cdot \mathrm{m}^{-3}$ 和 $22.50 \mathrm{~g} \cdot \mathrm{m}^{-3}$, 随土层 深度增加而不断降低(图1)。CK、LN、HN处理下0$45 \mathrm{~cm}$ 土层细根生物量分别为 $128.30 \mathrm{~g} \cdot \mathrm{m}^{-3}$ 、 $125.91 \mathrm{~g} \cdot \mathrm{m}^{-3} 、 143.87 \mathrm{~g} \cdot \mathrm{m}^{-3}$, 无显著差异。

\section{3 氮添加对扁刺栲细根寿命的影响}

扁刺栲细根的平均寿命与土层深度有关, 表现 为: I层> III层> II层。对照处理中, I、II、III层细根 的平均寿命分别为: 186、113、130天(表4)。生存曲 线的对数秩检验结果表明, 3 个土层中氮添加处理对 细根寿命的影响均未达到统计学显著水平(图2)。 Pearson相关分析表明, I层土壤扁刺栲细根的中值寿 命与表层土壤的 $\mathrm{pH}$ 值、根段表面积呈显著正相关关 系(表3)。

\section{4 氮添加对扁刺栲细根形态的影响}

在18个月的观察期内, 共观察到1 179条根(直 径 $<1 \mathrm{~mm}$, 均属细根范畴), 其中I层548条, II层 459 条, III层172条。I、II、III层扁刺栲根系的直径分别 为 $(0.86 \pm 0.13) \mathrm{mm} 、(0.61 \pm 0.01) \mathrm{mm}$ 和 $(0.67 \pm$ 0.06) $\mathrm{mm}$ 。与CK相比, LN处理对I层细根根段表面积 的影响达到了显著水平 $(p=0.049$, 降低了 $48.8 \%)$ 。

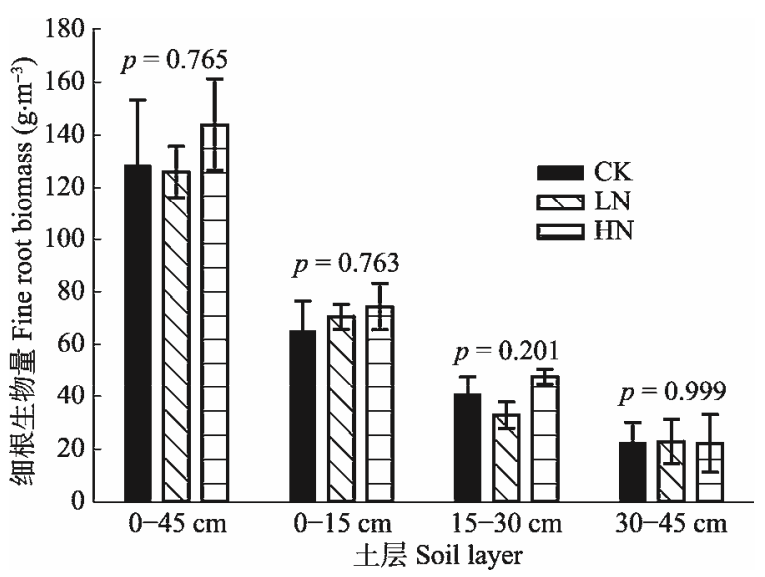

图1 不同土层细根生物量(平均值土标准误差)。CK, 对照 $\left(0 \mathrm{~g} \cdot \mathrm{m}^{-2} \cdot \mathrm{a}^{-1}\right) ; \mathrm{LN}$, 低氮 $\left(5 \mathrm{~g} \cdot \mathrm{m}^{-2} \cdot \mathrm{a}^{-1}\right) ; \mathrm{HN}$, 高氮 $\left(15 \mathrm{~g} \cdot \mathrm{m}^{-2} \cdot \mathrm{a}^{-1}\right) \circ p$ 值为不同土层单因素方差分析的结果。

Fig. 1 Fine root biomass in different soil layer (mean $\pm S E$ ). CK, control $\left(0 \mathrm{~g} \cdot \mathrm{m}^{-2} \cdot \mathrm{a}^{-1}\right)$; LN, low nitrogen addition $\left(5 \mathrm{~g} \cdot \mathrm{m}^{-2} \cdot \mathrm{a}^{-1}\right)$; $\mathrm{HN}$, high nitrogen addition $\left(15 \mathrm{~g} \cdot \mathrm{m}^{-2} \cdot \mathrm{a}^{-1}\right) . p$ value is the results of one-way ANOVA analysis in different soil layer.

氮添加未显著影响各土层扁刺栲根段长、根直径。 相关分析结果表明, I层扁刺栲根段表面积与土壤 $\mathrm{pH}$ 值呈显著的正相关关系(表3)。

\section{3 讨论}

\section{1 氮添加对土壤无机氮含量和土壤 $\mathrm{pH}$ 值的影响} 氮添加处理1.5年后, HN处理显著降低了表层 土壤 $\mathrm{pH}$ 值, 土壤硝态氮含量表现出随氮添加梯度增

表3 I 层 $(0-15 \mathrm{~cm})$ 扁刺栲细根形态指标、中值寿命和表层土壤 $(0-10 \mathrm{~cm})$ 理化指标相关分析结果

Table 3 Layer I $(0-15 \mathrm{~cm})$ correlation analysis results of Castanopsis platyacantha fine root morphology index, median lifespan, and soil physicochemical property $(0-10 \mathrm{~cm})$

\begin{tabular}{|c|c|c|c|c|c|c|c|}
\hline & $\begin{array}{c}\text { 中值寿命 } \\
\text { Median lifespan }\end{array}$ & $\mathrm{pH}$ & $\begin{array}{c}\text { 总氮 } \\
\text { Total nitrogen }\end{array}$ & $\mathrm{NH}_{4}^{+}-\mathrm{N}$ & $\mathrm{NO}_{3}^{-}-\mathrm{N}$ & $\begin{array}{c}\text { 根段长 } \\
\text { Root segment length } \\
\end{array}$ & $\begin{array}{c}\text { 根段表面积 } \\
\text { Root segment surface-area }\end{array}$ \\
\hline $\mathrm{pH}$ & $0.84^{* *}$ & & & & & & \\
\hline 总氮 Total nitrogen & -0.54 & -0.57 & & & & & \\
\hline $\mathrm{NH}_{4}^{+}-\mathrm{N}$ & -0.34 & $-0.75^{*}$ & 0.45 & & & & \\
\hline $\mathrm{NO}_{3}^{-}-\mathrm{N}$ & -0.41 & $-0.67^{*}$ & 0.54 & $0.72^{*}$ & & & \\
\hline 根段长 Root segment length & 0.37 & 0.35 & -0.15 & 0.01 & -0.18 & & \\
\hline 根段表面积 Root segment surface-area & $0.73^{*}$ & $0.74^{*}$ & -0.50 & -0.24 & -0.50 & $0.76^{*}$ & \\
\hline 根直径 Root diameter & 0.48 & 0.47 & -0.32 & -0.27 & -0.14 & -0.48 & 0.15 \\
\hline
\end{tabular}

加粗数字表示统计结果显著。

Bold figures show the results are statistical significant. *, $p<0.05$; **, $p<0.01$

表4 氮添加对扁刺栲细根平均寿命和中值寿命的影响(平均值土标准误差)

Table 4 Effects of $\mathrm{N}$ addition on Castanopsis platyacantha fine root average-lifespan and median-lifespan (mean $\pm S E$ )

\begin{tabular}{|c|c|c|c|c|c|c|}
\hline & \multicolumn{2}{|c|}{ 第I层 Layer I } & \multicolumn{2}{|c|}{ 第II层 Layer II } & \multicolumn{2}{|c|}{ 第III层 Layer III } \\
\hline & $\begin{array}{c}\text { 平均寿命 } \\
\text { Average lifespan (d) }\end{array}$ & $\begin{array}{c}\text { 中值寿命 } \\
\text { Median lifespan(d) }\end{array}$ & $\begin{array}{c}\text { 平均寿命 } \\
\text { Average lifespan (d) }\end{array}$ & $\begin{array}{c}\text { 中值寿命 } \\
\text { Median lifespan (d) }\end{array}$ & $\begin{array}{c}\text { 平均寿命 } \\
\text { Average lifespan (d) }\end{array}$ & $\begin{array}{c}\text { 中值寿命 } \\
\text { Median lifespan (d) }\end{array}$ \\
\hline CK & $186 \pm 9$ & $151 \pm 9$ & $113 \pm 8$ & $87 \pm 1$ & $130 \pm 13$ & $87 \pm 7$ \\
\hline $\mathrm{LN}$ & $181 \pm 30$ & $115 \pm 1$ & $101 \pm 8$ & $78 \pm 3$ & $171 \pm 20$ & $115 \pm 26$ \\
\hline $\mathrm{HN}$ & $168 \pm 9$ & $115 \pm 10$ & $113 \pm 8$ & $87 \pm 5$ & $158 \pm 18$ & $87 \pm 4$ \\
\hline
\end{tabular}

$\mathrm{CK}$, 对照; LN, 低氮; HN, 高氮。

$\mathrm{CK}$, control; LN, low nitrogen; HN, high nitrogen. 


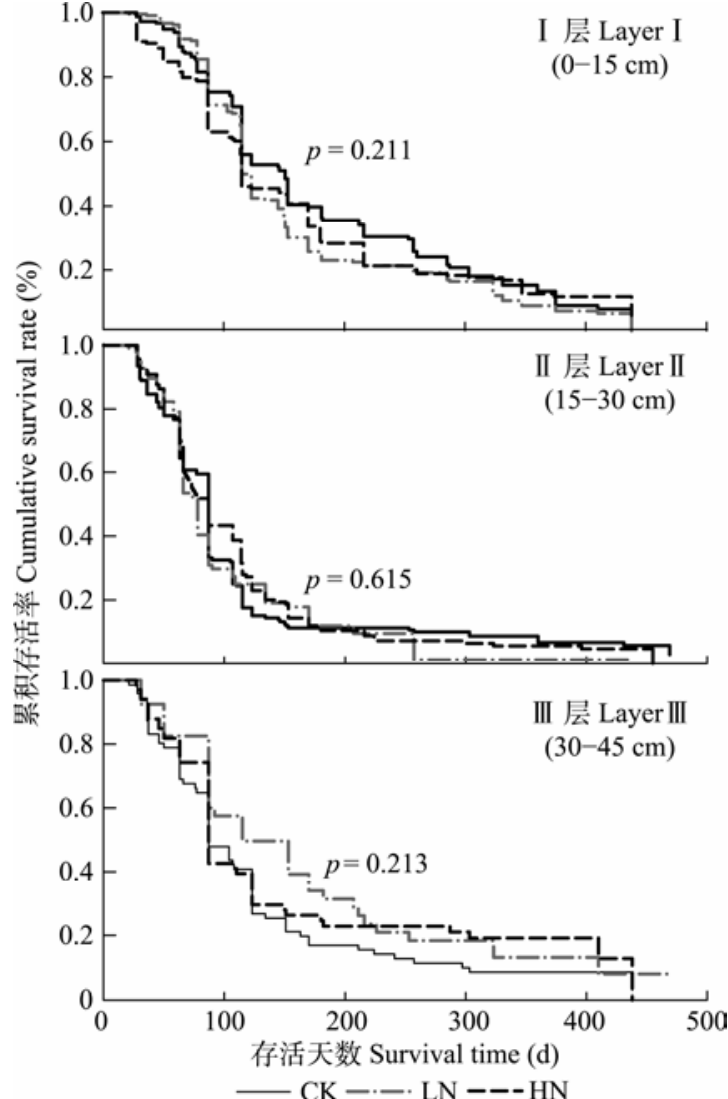

图2 氮添加对不同土层细根生存曲线的影响。p值为氮添加 处理对同一土层细根生存曲线的显著性检验结果。CK, 对照; $\mathrm{LN}$ ，低氮; HN，高氮。

Fig. 2 Effects of nitrogen additions on fine root survival curve. $p$ values are the survival curves significance test result of $\mathrm{N}$ addition in the same soil layers. CK, control; LN, low nitrogen; $\mathrm{HN}$, high nitrogen.

加而增加的趋势。土壤 $\mathrm{pH}$ 值与土壤 $\mathrm{NH}_{4}^{+}-\mathrm{N} 、 \mathrm{NO}_{3}^{-}-\mathrm{N}$ 含量呈显著负相关关系, 这与众多氮添加试验中的 发现一致(Huang et al., 2014)。一方面, 当 $\mathrm{NH}_{4}^{+}$被植 $^{-}$ 物根系所吸收时, 一个 $\mathrm{H}^{+}$将会释放到土壤溶液中, 增加土壤溶液酸度。另一方面, 当土壤中的无机氮 含量增加, 特别是超过了植物和微生物的需求时, 土壤中的硝化作用则会加强, 其结果使得土壤溶液 中的 $\mathrm{H}^{+}$增加(Evans et al., 2008)。 $\mathrm{H}^{+}$由于具有较强的 阳离子交换能力, 可将 $\mathrm{Na}^{+} 、 \mathrm{~K}^{+} 、 \mathrm{Mg}^{2+} 、 \mathrm{Ca}^{2+}$ 等盐基 阳离子从胶体颗粒中交换到土壤溶液中。这一过程 加速了湿润地区土壤盐基离子通过淋溶作用的损失 (Lucas et al., 2011), 并导致土壤对酸的缓冲能力减 弱(Tian \& Niu, 2015)。在本研究区域, 由于背景氮 沉降量很高, 达到 $90 \mathrm{~kg} \cdot \mathrm{hm}^{-2} \cdot \mathrm{a}^{-1}$ (Xu et al., 2013)。 进一步的氮添加处理(尤其是高氮处理)使得无机氮 含量接近或超过了植物和微生物的需求, 可能导致 了土壤接近或超过氮饱和，因此在高氮处理后几年
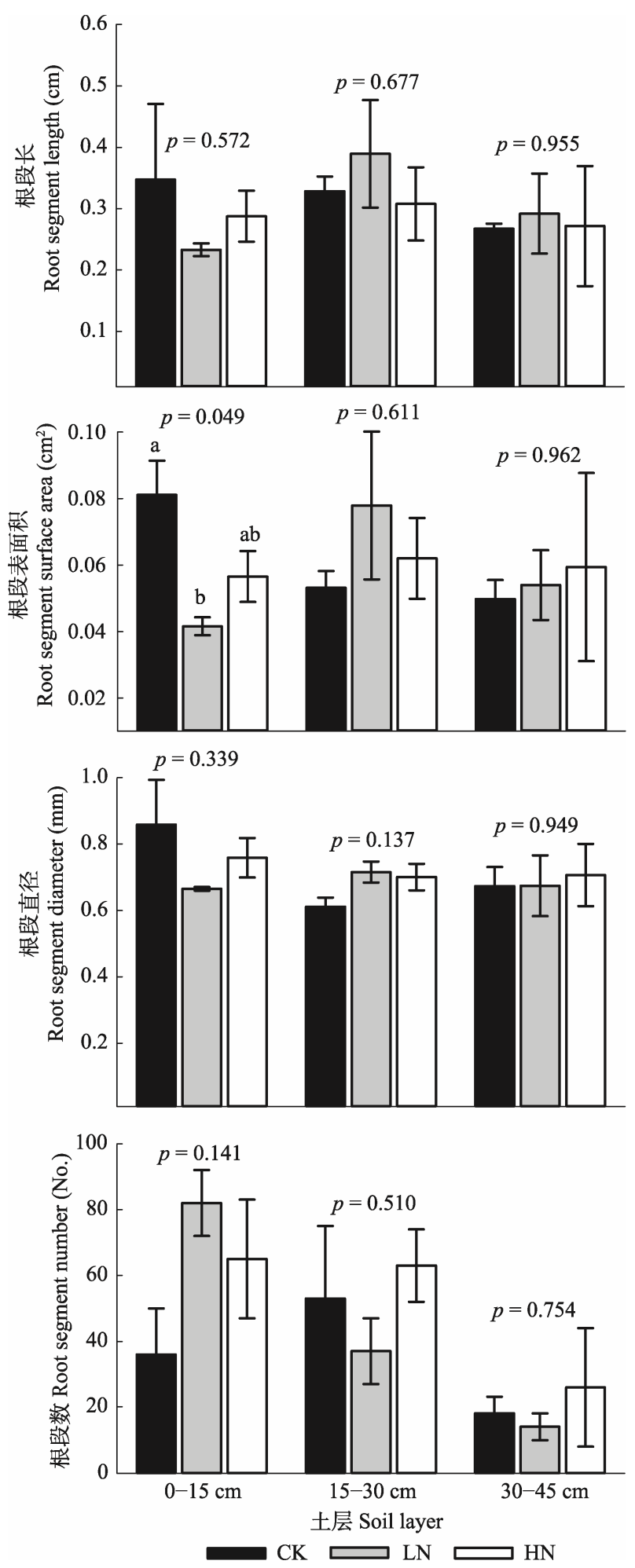

图3 氮添加对不同土层细根直径、根段数、根段长、根段 表面积的影响(平均值土标准误差)。CK, 对照; LN, 低氮; HN, 高氮。图中文字为对应土层的单因素方差分析结果; 图中字 母表示氮添加处理对同一土层各形态指标的单因素方差分 析结果。

Fig. 3 Effects of nitrogen additions on fine root diameter, length and superficial area at different soil layers (mean $\pm S E$ ). CK, control; LN, low nitrogen; HN, high nitrogen. Text and letters in figure are the result of one-way ANOVA analysis on same morphology indexes in the same soil layers. 
内就观察到土壤pH值显著降低。有较多研究观察到 氮添加导致土壤酸化, 并且往往伴随着土壤盐基离 子的损失, 严重的会导致土壤中 $\mathrm{Al}^{3+}$ 等金属离子活 化(Lucas et al., 2011)。例如, 本课题组前期研究发 现，持续每月一次的氮添加试验 6 年后，苦竹 (Pleioblastus amarus)人工林土壤交换性 $\mathrm{Al}$ 含量显著 增加, 其中高氮处理下增幅达 70\% (Chen et al., 2017)。而土壤酸化和金属离子活化会对物种多样 性、植被生产力、森林健康、土壤生物群落造成严 重影响(肖辉林, 2001)。

\section{2 氮添加对扁刺栲细根寿命的影响}

细根寿命由植物的本身特性包括根形态、分布 深度、菌根共生、植物生命形态等和外在特性包括 土壤氮和水分可利用性、温度等因素共同控制, 因 此不同树种及同一树种生长在不同环境中均表现出 不同的根寿命(Chen \& Brassard, 2013)。本研究结果 表明 $\mathrm{I}$ 层扁刺栲根系的平均寿命和中值寿命分别为 186天和151天。与中亚热带区域的树种相比, 扁刺 栲细根中值寿命低于常绿阔叶树种细柄罩树 (Altingia gracilipes, 184 天) 和米槠 (Castanopsis carlesii, 212天)的中值寿命, 以及针叶树种杉木 (Cunninghamia lanceolata, 236天)的中值寿命(袁一 丁等, 2009; 黄锦学等, 2012); 高于闽楠(Phoebe bournei, 89天)的中值寿命(郑金兴等, 2012)。

在本研究中, 虽然低氮和高氮处理均使 $\mathrm{I}$ 层扁 刺栲细根的中值寿命降低了 $24 \%$, 但是未达到统计 学显著水平。传统理论认为, 土壤氮可利用性增加, 通常提高植物地上部分的生长速率, 进而对养分元 素的需求也相应增加。植物为了支持地上部分对养 分的需求, 需要提高细根对土壤养分的吸收能力, 而新鲜的细根具有更强的养分获取能力, 因此植物 会提高根系统中年轻细根的比例, 降低细根的寿命, 加快整个根系统的周转速率(Eissenstat \& Yanai, 1997)。因此, 氮添加可能通过降低扁刺栲根系的中 值寿命, 加快根系周转速率以提高其吸收能力。

但是, 氮添加试验(Mei et al., 2010)和背景氮梯 度研究结果(Burton et al., 2000)并不总支持这一理 论。细根对氮素添加的不同响应可能归因于两方面。 首先是区域土壤养分可利用性差异(Chen \& Brassard, 2013)。当土壤氮限制比较严重, 长寿命的细根 可能只能给予植物较少的养分回报, 这时当土壤氮 可利用性提高时, 可能会极大地改善目前细根所处
环境的养分状况, 植物提高细根寿命对植物养分获 取更加有利。而土壤氮限制较小的状况下, 氮添加 可能会促进根系向根寿命更短的方向转变, 因为与 维持根系的碳投入相比, 发育新的细根可能对植物 更为有利。其次是树种差异。叶和根经济谱的研究 表明不同的树种具有不同的资源获取策略，有的树 种采取“快速投资-收益”策略，有的树种采取“保守 投资-收益”策略。不同的策略在适应土壤环境和养 分含量变化时, 会有不同的表现(陈䒯婷和许振柱, 2014; Weemstra et al., 2016)。于水强等(2009)同时研 究了水曲柳 (Fraxinus mandschurica) 和落叶松 (Larix gmelinii)细根寿命对土壤氮添加的响应, 结果 表明水曲柳细根中值寿命与土壤氮可利用性表现出 正相关关系, 而落叶松细根中值寿命对土壤氮可利 用性的变化没有显著响应。因此, 土壤初始养分状 况和树种对于细根寿命对氮添加的响应有重要的影 响。对于本研究试验样地, 由于土壤氮素含量较高, 并且具有很高的背景氮沉降量。区域天然林植被可 能处于氮饱和状态, 因此进一步的氮添加并未对扁 刺栲的根系养分吸收策略产生显著影响, 导致氮添 加未对扁刺栲根寿命产生显著影响。

\section{3 氮添加对扁刺栲细根生物量和形态特征的影响}

氮添加处理未影响3个土层中扁刺栲的细根生 物量。目前, 氮添加对细根生物量的影响存在减少、 增加、不影响3种情况(Wang et al., 2012; Noguchi et al., 2013; Kou et al., 2015)。细根生物量对氮添加的 不同响应方向可能与不同气候区域有关。Li等(2015) 整合全球相关研究表明, 氮添加显著增加了北方森 林的细根生物量, 而没有改变热带、亚热带森林的 细根生物量, 分析这可能与区域土壤养分状况有关; 另外氮添加对细根生物量的影响也与氮添加量以及 氮处理时间有关。

在本研究中, 氮添加使I层扁刺栲细根的根段 长和根段表面积呈下降趋势, 其中低氮处理对根段 表面积的影响达到显著 $(p=0.049)$ 水平。植物依靠根 系获取自身需要的多种元素, 由于土壤各种养分具 有较强的空间异质性。植物根系利用其强大的可塑 性予以应对, 形成了“成本-效益”策略来适应各种土 壤养分分布状况(Bardgett et al., 2014)。然而在本研 究中, 虽然氮添加使表层土壤的无机氮增加了 $8.6 \%-9.2 \%$, 但相关分析结果表明 $\mathrm{I}$ 层扁刺栲根形态 指标与表层土壤 $\mathrm{NH}_{4}^{+}-\mathrm{N} 、 \mathrm{NO}_{3}^{-}-\mathrm{N}$ 含量之间并无显著 
的相关关系，而根段表面积与土壤 $\mathrm{pH}$ 值之间存在显 著的正相关关系。这说明在该次生常绿阔叶林中, 与无机氮可利用性相比, 扁刺栲形态特征与土壤 $\mathrm{pH}$ 值有更为紧密的关系。

\section{4 结论}

高氮处理显著降低了该次生林表层土壤 $\mathrm{pH}$ 值, 低氮处理显著降低了 0-15 cm土层扁刺栲细根的根 段表面积。根段表面积与土壤 $\mathrm{pH}$ 值之间存在显著的 正相关关系。氮添加处理未影响扁刺栲细根寿命和 生物量。本研究表明氮添加不会影响扁刺栲细根的 寿命和生物量。仅可能通过改变该次生林表层土壤 无机氮含量, 导致土壤一定程度的酸化, 进而对表 层土壤的扁刺栲根系形态特征造成一定的影响。

\section{基金项目 国家自然科学基金(31300522)。}

\section{参考文献}

Addo-Danso SD, Prescott CE, Smith AR (2016). Methods for estimating root biomass and production in forest and woodland ecosystem carbon studies: A review. Forest Ecology and Management, 359, 332-351.

Balogianni VG, Blume-Werry G, Wilson SD (2016). Root production in contrasting ecosystems: The impact of rhizotron sampling frequency. Plant Ecology, 217, 1-9.

Bao SD (2000). Soil Agricultural Chemistry Analysis. 3rd edn. China Agriculture Press, Beijing. (in Chinese) [鲍士旦 (2000). 土壤农化分析. 第三版. 中国农业出版社, 北京.]

Bardgett RD, Mommer L, de Vries FT (2014). Going underground: Root traits as drivers of ecosystem processes. Trends in Ecology \& Evolution, 29, 692-699.

Bedison JE, Mcneil BE (2009). Is the growth of temperate forest trees enhanced along an ambient nitrogen deposition gradient? Ecology, 90, 1736-1742.

Burton AJ, Pregitzer KS, Hendrick RL (2000). Relationships between fine root dynamics and nitrogen availability in Michigan northern hardwood forests. Oecologia, 125, 389-399.

Chen GT, Tu LH, Peng Y, Hu HL, Hu TX, Xu ZF, Liu L, Tang $Y$ (2017). Effect of nitrogen additions on root morphology and chemistry in a subtropical bamboo forest. Plant and Soil, 412, 441-451.

Chen HYH, Brassard BW (2013). Intrinsic and extrinsic controls of fine root life span. Critical Reviews in Plant Sciences, 32, 151-161.

Chen YT, XU ZZ (2014). Review on research of leaf economics spectrum. Chinese Journal of Plant Ecology, 38, 1135-1153. (in Chinese with English abstract) [陈荣婷,
许振柱 (2014). 植物叶经济谱的研究进展. 植物生态学 报, 38, 1135-1153.]

Chirici G, Corona P, Portoghesi L (2013). Global forest resources assessment. Italian Journal of Forest \& Mountain Environments, 56, 12-64.

Eissenstat DM, Wells CE, Yanai RD, Whitbeck JL (2000). Building roots in a changing environment: Implications for root longevity. New Phytologist, 147, 33-42.

Eissenstat DM, Yanai RD (1997). The ecology of root lifespan. Advances in Ecological Research, 27, 1-60.

Evans CD, Norris D, Ostle N, Grant H, Rowe EC, Curtis CJ, Reynolds B (2008). Rapid immobilisation and leaching of wet-deposited nitrate in upland organic soils. Environment Pollution, 156, 313-319.

Galloway JN, Cowling EB (2002). Reactive nitrogen and the world: 200 years of change. Ambio, 31, 64-71.

Galloway JN, Dentener FJ, Capone DG, Boyer EW, Howarth RW, Seitzinger SP, Asner GP, Cleveland CC, Green PA, Holland EA, Karl DM, Michaels AF, Porter JH, Townsend AR, Vörösmarty CJ (2004). Nitrogen cycles: Past, present, and future. Biogeochemistry, 70, 153-226.

Hou X, Duan C, Tang CQ, Fu D (2010). Nutrient relocation, hydrological functions, and soil chemistry in plantations as compared to natural forests in central Yunnan, China. Ecological Research, 25, 139-148.

Huang J, Mo J, Zhang W, Lu X (2014). Research on acidfication in forest soil driven by atmospheric nitrogen deposition. Acta Ecologica Sinica, 34, 302-310.

Huang JX, Ling H, Yang ZJ, Lu ZL, Xiong DC, Chen GS, Yang YS, Xie JS (2012). Estimating fine root production and mortality in subtropical Altingia grlilipes and Castanopsis carlesii forests. Acta Pedologica Sinica, 14, 4472-4480. (in Chinese with English abstract) [黄锦学, 凌华, 杨智杰, 卢正立, 熊德成, 陈光水, 杨玉盛, 谢锦 升 (2012). 中亚热带细柄阿丁枫和米槠群落细根的生 产和死亡动态. 生态学报, 14, 4472-4480.]

Jackson RB, Mooney HA, Schulze ED (1997). A global budget for fine root biomass, surface area, and nutrient contents. Proceedings of the National Academy of Sciences of the United States of America, 94, 7362-7366.

Johnson MG, Phillips DL, Tingey DT, Storm MJ (2000). Effects of elevated $\mathrm{CO}_{2}$, N-fertilization, and season on survival of ponderosa pine fine roots. Canadian Journal of Forest Research, 30, 220-228.

Jourdan C, Silva EV, Goncalves JLM, Ranger J, Moreira RM, Laclau JP (2008). Fine root production and turnover in Brazilian Eucalyptus plantations under contrasting nitrogen fertilization regimes. Forest Ecology and Management, 256, 396-404.

Kou L, Guo D, Yang H, Gao W, Li S (2015). Growth, morphological traits and mycorrhizal colonization of fine roots respond differently to nitrogen addition in a slash pine

www.plant-ecology.com 
plantation in subtropical China. Plant and Soil, 391, 207-218.

Lebauer DS, Treseder KK (2008). Nitrogen limitation of net primary productivity in terrestrial ecosystems is globally distributed. Ecology, 89, 371-379.

Li D, Chen HW, Li GQ, Yang B, Meng M, Shi FQ (2011). Comparative analysis of artificial forest and natural forest in China. Forest Inventory and Planning, 36(6), 59-63. (in Chinese with English abstract) [李丹, 陈宏伟, 李根前, 杨斌, 孟梦, 史富强 (2011). 我国天然林与人工林的比 较研究. 林业调查规划, 36(6), 59-63.]

Li JY, Wang MB, Shi JW (2007). Minirhizotron technique in measuring fine root indices: A review. Chinese Journal of Ecology, 26, 1842-1848. (in Chinese with English abstract) [李俊英, 王孟本, 史建伟 (2007). 应用微根管法 测定细根指标方法评述. 生态学杂志, 26, 1842-1848.]

Li W, Jin C, Guan D, Wang Q, Wang A, Yuan F, Wu J (2015). The effects of simulated nitrogen deposition on plant root traits: A meta-analysis. Soil Biology and Biochemistry, 82, 112-118.

Lucas RW, Klaminder J, Futter MN, Bishop KH, Egnell G, Laudon H, Hogberg P (2011). A meta-analysis of the effects of nitrogen additions on base cations: Implications for plants, soils, and streams. Forest Ecology and Management, 262, 95-104.

Lü CQ, Tian HQ, Huang Y (2007). Ecological effects of increased nitrogen deposition in terrestrial ecosystems. Journal of Plant Ecology (Chinese Version), 31, 205-218. (in Chinese with English abstract) [吕超群, 田汉勤, 黄耀 (2007). 陆地生态系统氮沉降增加的生态效应. 植物生 态学报, 31, 205-218.]

Majdi H, Pregitzer K, Moren A, Nylund J, Gren GRIA (2005). Measuring fine root turnover in forest ecosystems. Plant and Soil, 276, 1-8.

Mei L, Gu J, Zhang Z, Wang Z (2010). Responses of fine root mass, length, production and turnover to soil nitrogen fertilization in Larix gmelinii and Fraxinus mandshurica forests in Northeastern China. Journal of Forest Research, 15, 194-201.

Nacry P, Bouguyon E, Gojon A (2013). Nitrogen acquisition by roots: Physiological and developmental mechanisms ensuring plant adaptation to a fluctuating resource. Plant and Soil, 370, 1-29.

Noguchi K, Nagakura J, Kaneko S (2013). Biomass and morphology of fine roots of sugi (Cryptomeria japonica) after 3 years of nitrogen fertilization. Frontiers in Plant Science, $4,1-7$.

Ostonen I, Lohmus K, Helmisaari HS, Truu J, Meel S (2007). Fine root morphological adaptations in scots pine, Norway spruce and silver birch along a latitudinal gradient in boreal forests. Tree Physiologist, 27, 1627-1634.

Poorter H, Niklas KJ, Reich PB, Oleksyn J, Poot P, Mommer L
(2011). Biomass allocation to leaves, stems and roots: Meta-analyses of interspecific variation and environmental control. New Phytologist, 193, 30-50.

Shi JW, Yu SQ, Yu LZ, Han YZ, Wang ZQ, Guo DL (2006). Application of minirhizotron in fine root studies. Chinese Journal of Applied Ecology, 17, 715-719. (in Chinese with English abstract) [史建伟, 于水强, 于立忠, 韩有志, 王 政权, 郭大立 (2006). 微根管在细根研究中的应用. 应 用生态学报, 17, 715-719.]

Song G, Sun B, Jiao JY (2007). Comparison between ultraviolet spectrophotometry and other methods in determination of soil nitrate-N. Acta Pedologica Sinica, 44, 288-293. (in Chinese with English abstract) [宋歌, 孙波, 教剑英 (2007). 测定土壤硝态氮的紫外分光光度法与其他方法 的比较. 土壤学报, 44, 288-293.]

Tian D, Niu S (2015). A global analysis of soil acidification caused by nitrogen addition. Environmental Research Letter, 10, 1-10.

Tingey DT, Phillips DL, Johnson MG (2003). Optimizing minirhizotron sample frequency for an evergreen and deciduous tree species. New Phytologist, 157, 155-161.

Wang C, Han S, Zhou Y, Yan C, Cheng X, Zheng X, Li MH (2012). Responses of fine roots and soil $N$ availability to short-term nitrogen fertilization in a broad-leaved Korean pine mixed forest in northeastern China. PLOS ONE, 7, e31042. doi: 10.1371/journal.pone.0031042.

Weemstra M, Mommer L, Visser EJW, van Ruijven J, Kuyper TW, Mohren GMJ, Sterck FJ (2016). Towards a multidimensional root trait framework: A tree root review. New Phytologist, 211, 1159-1169.

Wu YB, Che RX, Ma S, Deng YC, Zhu MJ, Cui XY (2014). Estimation of root production and turnover in an alpine meadow: Comparison of three measurement methods. Acta Ecologica Sinica, 34, 3529-3537. (in Chinese with English abstract) [吴伊波, 车荣晓, 马双, 邓永翠, 朱敏 健, 崔骁勇 (2014). 高寒草甸植被细根生产和周转的比 较研究. 生态学报, 34, 3529-3537.]

Wurzburger N, Wright SJ (2015). Fine root responses to fertilization reveal multiple nutrient limitation in a lowland tropical forest. Ecology, 96, 2137-2146.

Xiao HL (2001). Effects of atmospheric nitrogen deposition on forest soil acidification. Scientia Silvae Sinica, 37(4), 111-116. (in Chinese with English abstract) [肖辉林 (2001). 大气氮沉降对森林土壤酸化的影响. 林业科学, 37(4), 111-116.]

Xu ZF, Tu LH, Hu TX (2013). Implications of greater than average increases in nitrogen deposition on the western edge of the Szechwan basin, China. Environmental Pollution, 177, 201-202.

Yu SQ, Wang ZQ, Shi JW, Yu LZ, Quan XQ (2009). Effects of nitrogen fertilization on fine root lifespan of Fraxinus mandshurica and Larixgme. Chinese Journal of Applied 
Ecology, 20, 2332-2338. (in Chinese with English abstract) [于水强, 王政权, 史建伟, 于立忠, 全先奎 (2009). 氮肥对水曲柳和落叶松细根寿命的影响. 应用 生态学报, 20, 2332-2338.]

Yuan YD, Yang YS, Chen GS, Yang ZJ, Lin RQ, Ling H (2009). Fine root longevity of a Cunninghamia lanceolata plantation estimated by minirhizotrons. Journal of Subtropical Resources and Environment, 4(2), 47-52. (in Chinese with English abstract) [袁一丁, 杨玉盛, 陈光水, 杨智杰, 林如强, 凌华 (2009). 杉木人工林细根寿命研
究. 亚热带资源与环境学报, 4(2), 47-52.]

Zheng JX, Huang JX, Wang ZZ, Xiong DC, Yang ZJ, Chen GS (2012). Fine root longevity and controlling factors in a Phoebe bournei plantation. Acta Ecologica Sinica, 23, 7532-7539. (in Chinese with English abstract) [郑金兴, 黄锦学, 王珍珍, 熊德成, 杨智杰, 陈光水 (2012). 闽 楠人工林细根寿命及其影响因素. 生态学报, 23, 7532-7539.]

责任编委: 贺金生 责任编辑: 李 敏

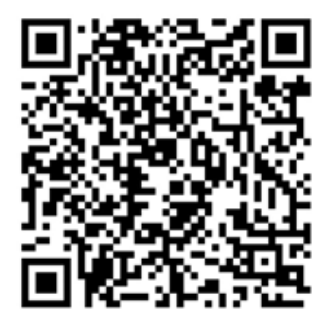

扫码向作者提问 